\title{
Simulação Multifásica de Abastecimento visando Avaliar o Impacto de Diferentes Pressões na Entrada do Tanque de Combustível de Veículos Automotivos
}

\author{
Filipe Fabian Buscariolo ${ }^{1.3}$, Felipe Magazoni ${ }^{1.2}$, Flavio Maruyama ${ }^{1}$, Julio Cesar Lelis Alves ${ }^{1}$ e \\ Leonardo D. Volpe ${ }^{1}$ \\ ${ }^{1} \mathrm{GM}$ Brasil \\ ${ }^{2} \mathrm{NETeF-USP}$ \\ ${ }^{3} \mathrm{NDF}-\mathrm{USP}$
}

E-mails: filipe.buscariolo@gm.com, felipe.magazoni@gm.com, flavio.maruyama@gm.com, julio.alves@gm.com, leonardo.volpe@gm.com

\section{RESUMO}

A geometria do duto de abastecimento impacta na velocidade e na qualidade do abastecimento de veículos. Quando o tanque se encontra totalmente vazio, a pressão interna está muito alta, e se o projeto do duto de abastecimento não é adequado, o fluido não consegue vencer esta pressão, podendo causar o retorno do combustível em direção à pistola, gerando até vazamento, ou também, o desligamento prematuro do processo. Os recentes avanços tanto nos códigos CFD quanto no poder computacional permitiram que simulações multifásicas fossem realizadas em nível de produção por indústrias mundo afora. Este trabalho leva em conta esses avanços e propõe uma metodologia para uma simulação de abastecimento de combustível multifásica utilizando CFD para avaliar dutos de combustível de forma que a pressão interna do tanque não venha a causar uma parada prematura no abastecimento nem vazamento de combustível. $\mathrm{O}$ trabalho avalia o escoamento do combustível, considerando dois valores fixos e uma curva de pressão do tanque de combustível em função do tempo, medida através de um teste físico, com o objetivo de avaliar a perda de carga e o tempo de abastecimento. A metodologia proposta foi capaz de reproduzir a atividade de abastecimento de combustível de um veículo, tornando possível avaliar diferentes configurações de dutos de abastecimento, quanto sua performance, antes que um protótipo seja construído.

\section{INTRODUÇÃO}

Com o constante crescimento do poder computacional, simulações CFD se tornaram uma boa solução para predizer o desempenho de mecânica dos fluidos. Este fator torna possível a simulação de problemas mais complexos, como por exemplo simulações multifásicas, envolvendo dois ou mais fluidos.

De acordo com BUSCARIOLO e VOLPE (2014) [3], simulações multifásicas CFD podem ser divididas em dois principais grupos:

- Reativo: fluidos interagem quimicamente ou fisicamente gerando novos produtos e alterando as propriedades termodinâmicas. Exemplo: combustão; 
- Não-reativo: dois ou mais fluidos estão presentes no domínio computacional, entretanto eles não se interagem quimicamente, somente fisicamente.

Este trabalho tem como objetivo contribuir para o crescimento das simulações multifásicas realizadas na indústria. O principal objetivo é propor uma metodologia para uma simulação de abastecimento de combustível multifásica utilizando CFD para avaliar dutos de combustível medindo o tempo total de abastecimento, antes que qualquer protótipo seja construído, ajudando assim para melhorar o desenvolvimento de veículos.

A geometria do duto de abastecimento impacta na velocidade e na qualidade do abastecimento de veículos. Quando o tanque se encontra totalmente vazio, a pressão interna está muito alta, e se o projeto do duto de abastecimento não é adequado, o fluido não consegue vencer esta pressão, podendo causar o retorno do combustível em direção à pistola, gerando até vazamento, ou também, o desligamento prematuro do processo.

BANERJEE et al. (2002) [1] propuseram um estudo sobre a dinâmica de alguns componentes críticos durante o processo de abastecimento de combustível para carros de passeio e caminhões leves. O duto de combustível, tubo de respiro do tanque, tanque e o canister foram investigados. A pressão do tanque, identificada com um parâmetro crítico no desempenho do fluxo de abastecimento, é medido e utilizado como condição de contorno. Experimentos foram realizados e utilizados para validar as simulações computacionais. As simulações indicaram que CFD pode ser utilizado como uma ferramenta de desenvolvimento e redução de custo do sistema de abastecimento de combustível.

Durante o desenvolvimento do sistema de abastecimento, a metodologia permite a avaliação de diversas geometrias de dutos e tanques de combustível, e problemas devido ao abastecimento podem ser evitados antes de qualquer protótipo físico.

\section{OBJETIVO}

Baseado nas condições reais do abastecimento em postos de combustível, uma vazão volumétrica de $50 \mathrm{l} / \mathrm{min}$ foi utilizada para que as condições de simulação virtual fossem reproduzidas. Um exemplo de um veículo sendo abastecido em postos de combustível está apresentado na Figura 1.

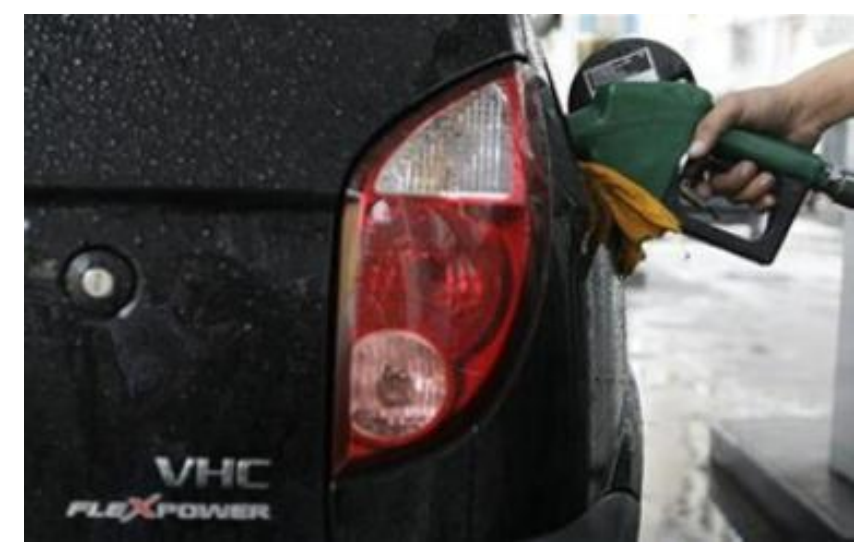

Figura 1. Veículo sendo abastecido 
O principal objetivo deste trabalho é avaliar o desenho do duto de combustível, medindo o tempo total de abastecimento antes que qualquer protótipo físico seja construído utilizando a metodologia computacional em CFD. A segunda parte propõe uma análise paramétrica da pressão do tanque, considerando dois valores fixos de $500 \mathrm{~Pa}$ e $1000 \mathrm{~Pa}$ e uma curva de pressão do tanque de combustível em função do tempo, medida através de um teste físico. Os valores fixos da pressão do tanque representam em média o tanque com metade de combustível (500 $\mathrm{Pa})$ e o tanque vazio $(1000 \mathrm{~Pa})$.

\section{METODOLOGIA}

A metodologia proposta neste trabalho é baseado em correlacionar a condição real com a simulação multifásica CFD proposta para o abastecimento de combustível. Esta metodologia é similar às propostas por BUSCARIOLO e VOLPE (2014) [3] e BUSCARIOLO, VOLPE e ALVES (2014) [4].

O sistema de abastecimento simulado no presente trabalho está apresentado na Figura 2. A vazão mássica é inserida na pistola da bomba de combustível a fim de avaliar o sistema de abastecimento. A análise considera somente o duto de combustível e a pistola da bomba.

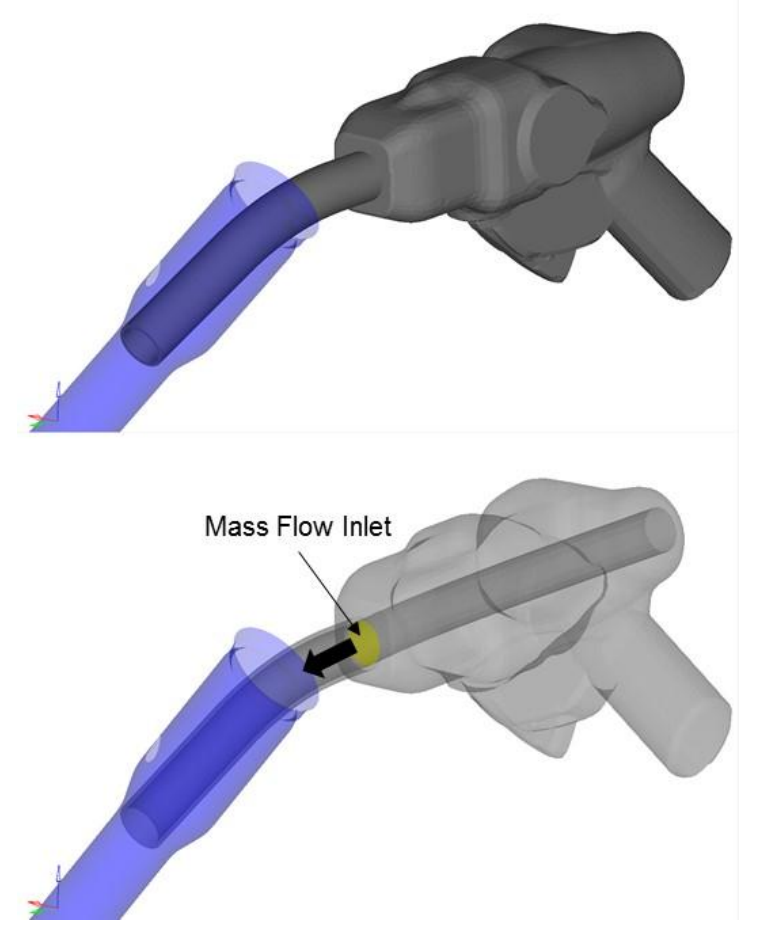

Figura 2. Pistola da bomba de combustível (início do abastecimento)

A pressão de entrada no duto de combustível é representada pela pressão ambiente, e a pressão de saída do duto é a mesma do tanque, aonde foram consideradas três condições operacionais:

1. Pressão do tanque constante, $500 \mathrm{~Pa}$;

2. Pressão do tanque constante, $1000 \mathrm{~Pa}$;

3. Curva de pressão do tanque x tempo de acordo com a Figura 3. 


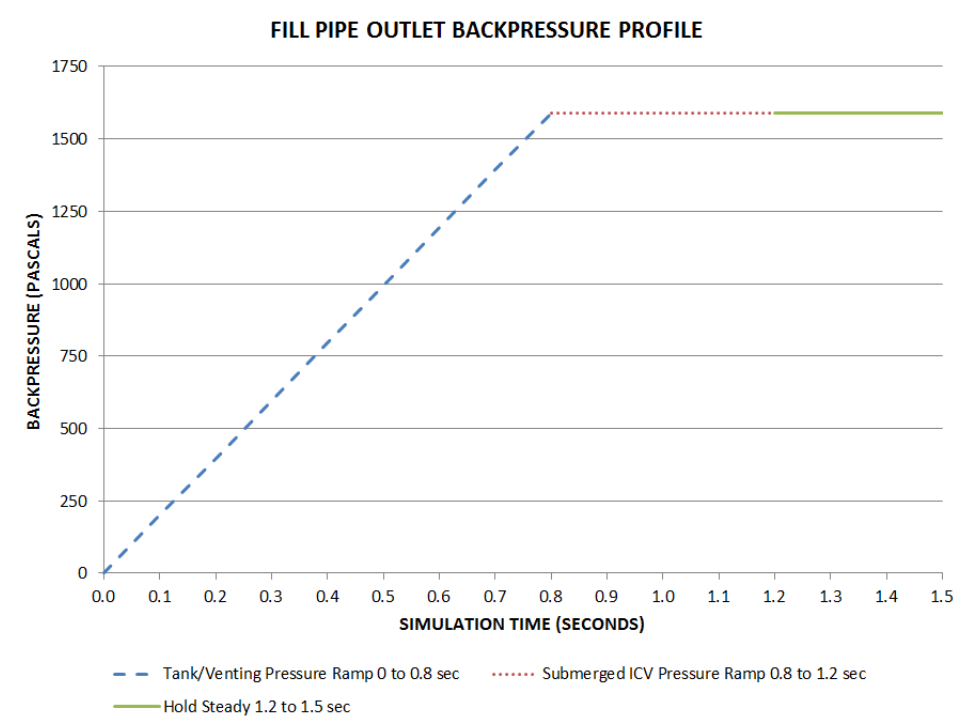

Figura 3. Perfil da pressão do tanque ao longo do tempo de abastecimento

Os parâmetros de simulação considerados neste trabalho estão apresentados na Tabela 1.

Tabela 1. Parâmetros utilizados nas simulações computacionais

\begin{tabular}{|c|c|}
\hline Vazão volumétrica & $501 / \mathrm{min}$ \\
\hline Pressão do tanque & Perfil da pressão do tanque \\
\hline Pressão de entrada do duto & Pressão ambiente \\
\hline Intensidade de turbulência & $0.60 \%$ \\
\hline Modelo de turbulência & $\mathrm{k}-\varepsilon$ \\
\hline
\end{tabular}

\section{CONFIGURAÇÃO DO MODELO}

Para a configuração do modelo multifásico, considera-se o método Volume of Fluid (VOF), que é uma técnica numérica para localizar a superfície livre. Ele pertence aos métodos eulerianos.

VOF utiliza um esquema de advecção, uma ferramenta numérica que permite que o programador localize o formato e a posição da interface entre os fluidos. As equações de Navier-Stokes descrevem o movimento do escoamento e precisam ser resolvidos separadamente.

O modelo de turbulência considerado é o k-E, que é um dos modelos mais comuns utilizados para simular condições turbulentas em CFD. É um modelo de duas equações. A primeira equação determina a energia cinética turbulenta, k. A segunda equação determina a taxa de dissipação turbulenta, $\varepsilon$.

A simulação foi configurada como sendo transiente com um passo de tempo pequeno o suficiente para que a simulação convirja e represente corretamente o escoamento. O tempo total simulado é calculado como sendo a razão entre o comprimento do duto e a velocidade do fluido (vazão volumétrica dividida pela área de entrada). 


\section{RESULTADOS}

A metodologia proposta e o modelo são capazes de reproduzir o escoamento multifásico de um veículo sendo abastecido com uma vazão volumétrica de 50 1/min.

A pressão de entrada do duto de combustível é ambiente e a pressão do tanque possui três condições operacionais:

1. Pressão do tanque constante, $500 \mathrm{~Pa}$;

2. Pressão do tanque constante, $1000 \mathrm{~Pa}$;

3. Curva de pressão do tanque x tempo de acordo com a Figura 3.

Analisa-se o escoamento próximo da entrada do duto de combustível no tempo de $0.2 \mathrm{~s}$, como apresentado na Figura 4. Com uma pressão do tanque mais alta (caso 2), o combustível retornou para a região da pistola, desligando a bomba e até gerando vazamento, uma vez que o fluido não consegue vencer a pressão interna do tanque.

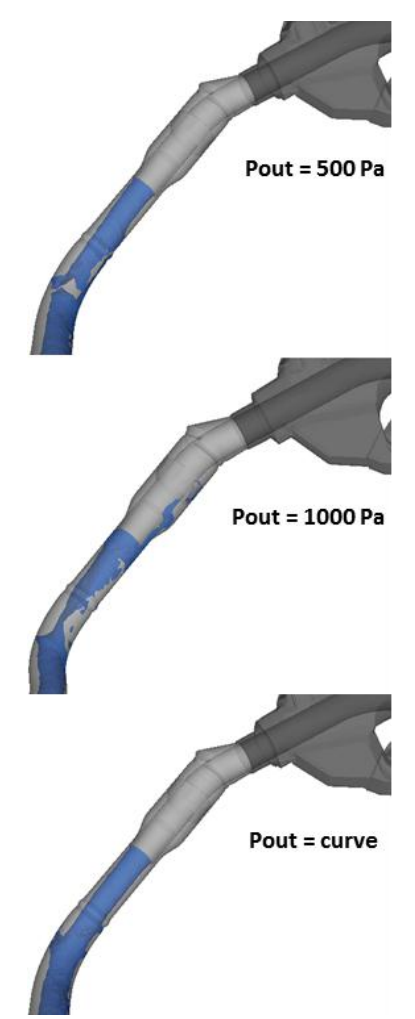

Figura 4. Detalhe do processo de abastecimento no tempo de $0.2 \mathrm{~s}$

O processo completo de abastecimento pode ser visto nas Figura 5, 6 e 7. Para os casos 1 e 3, não existe retorno de combustível. Considerando os casos com pressão fixa (casos 1 e 2), é possível concluir que quanto maior a pressão dentro do tanque de combustível, mais problemas com o abastecimento podem ocorrer, entretanto estas duas condições não representam o processo real. 
O caso 3 utiliza uma curva de pressão do tanque versus tempo medida através de testes físicos durante o processo de abastecimento, e os resultados podem ser visualizados na Figura 7.

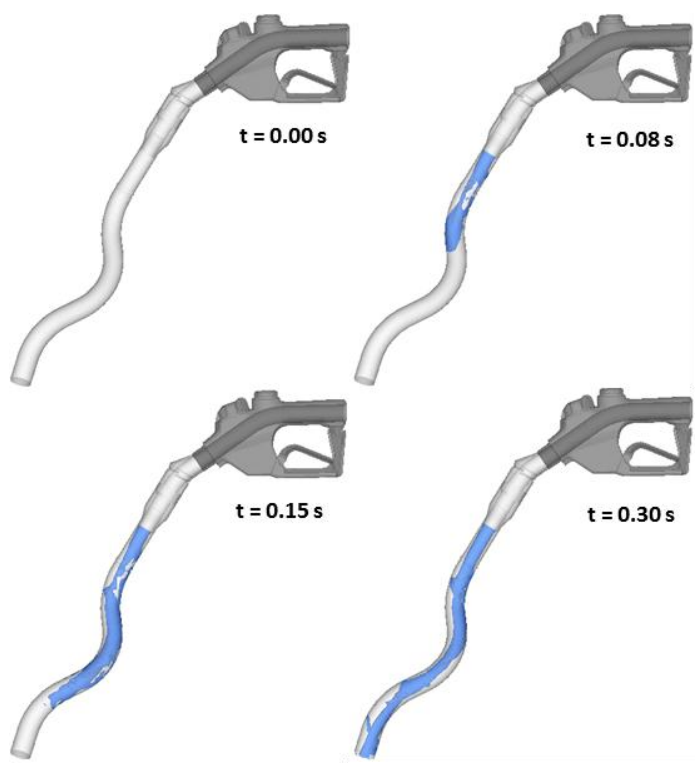

Figura 5. Escoamento dentro do duto de combustível com uma pressão do tanque de $500 \mathrm{~Pa}$

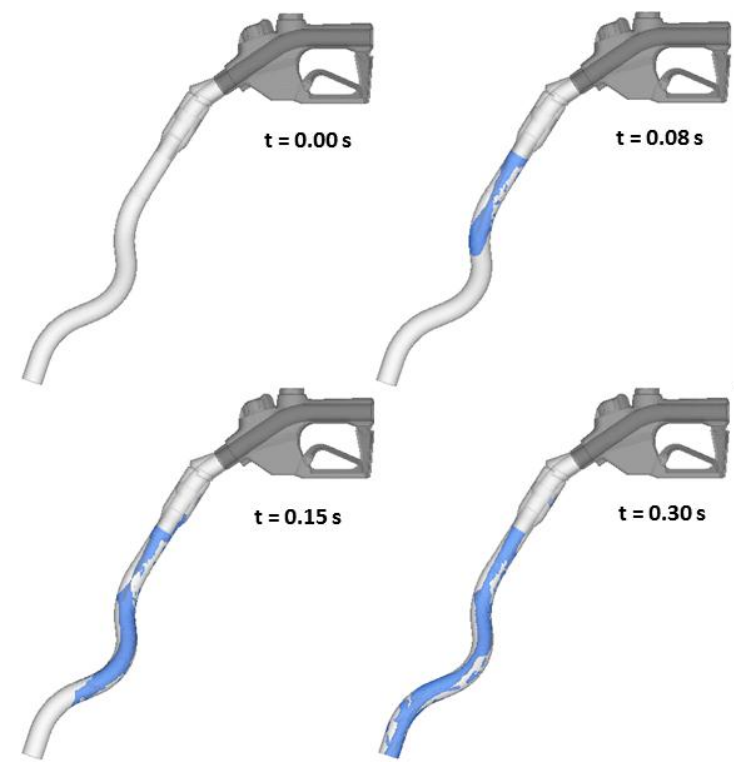

Figura 6. Escoamento dentro do duto de combustível com uma pressão do tanque de $1000 \mathrm{~Pa}$ 


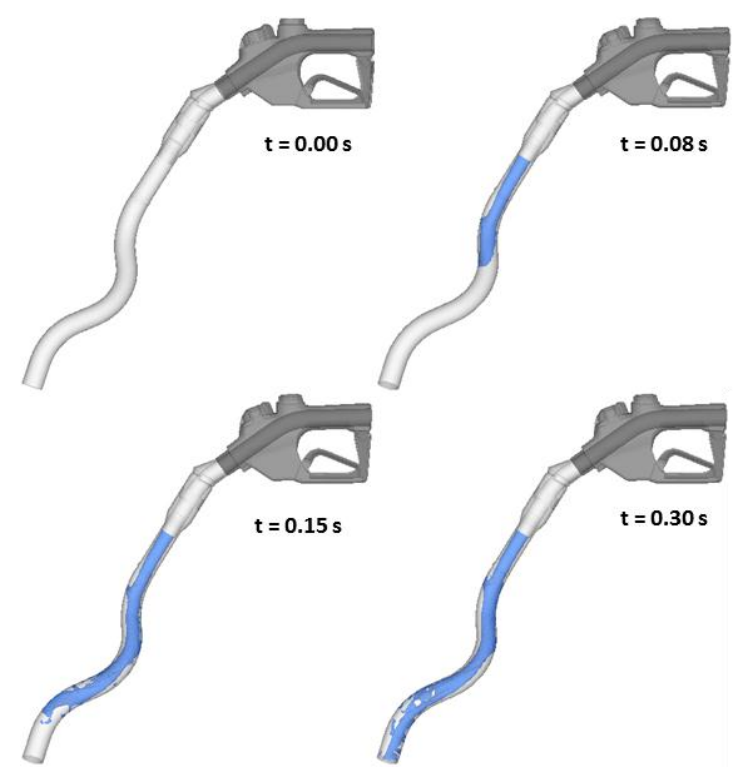

Figura 7. Escoamento dentro do duto de combustível com uma pressão do tanque variável

É possível concluir que o melhor caso para avaliar o processo de abastecimento de combustível é similar ao caso 3 com pressão do tanque variável, uma vez que isto representa o comportamento real de abastecimento de combustível no tanque.

A curva de perda de carga de combustível e ar entre a saída da pistola da bomba de combustível e a entrada do tanque para os três casos são apresentados na Figura 8. Estas variações influenciam o tempo de abastecimento, que é apresentado na Tabela 2. A simulação do caso 3 com pressão de tanque variável, que representa o processo real, mostra um resultado de até 0.25 s comparado aos outros casos e $65 \mathrm{~Pa}$ de perda de carga a mais que o caso 1.

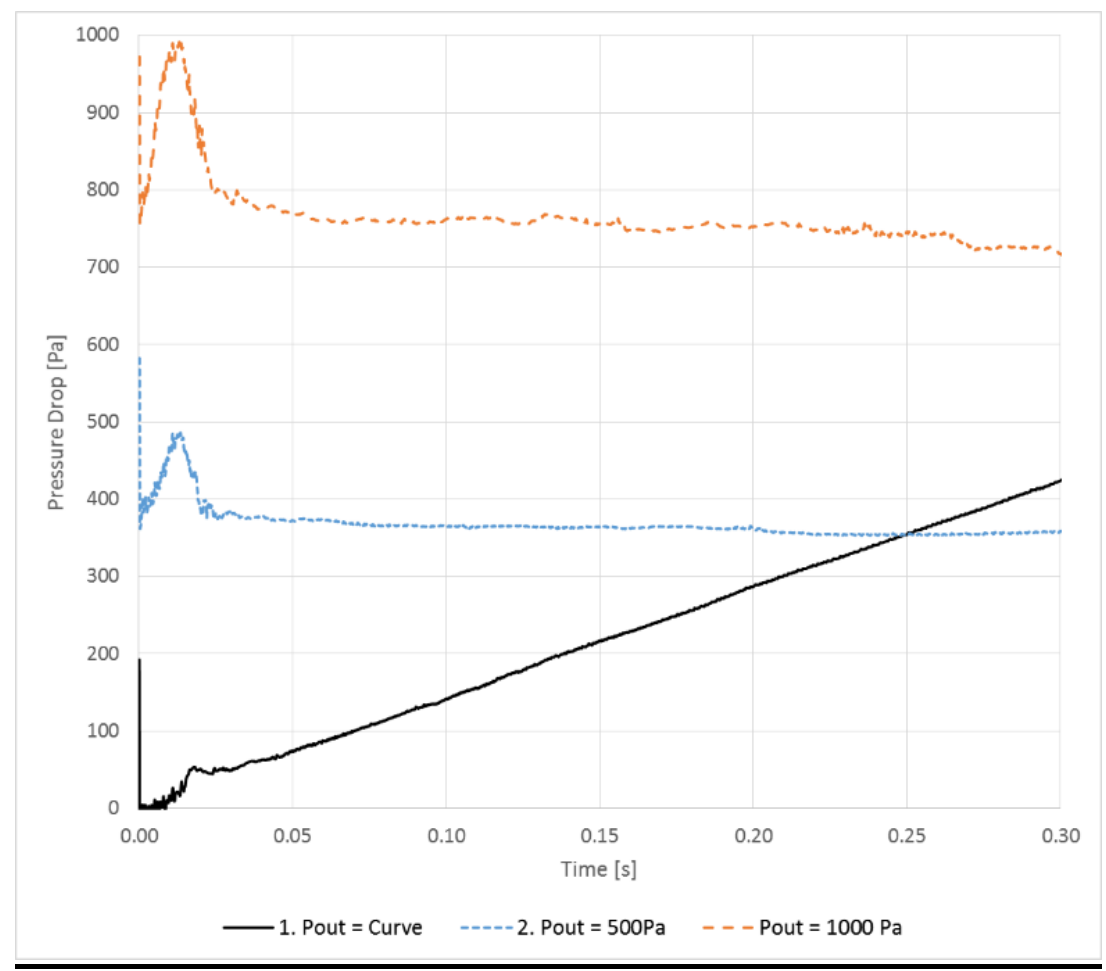

Figura 8. Perda de carga versus tempo para as três configurações 
Tabela 2. Tempo de abastecimento e perda de carga média para as três configurações

\begin{tabular}{|c|c|c|c|}
\hline Caso & $\begin{array}{c}\text { Pressão média } \\
\text { do tanque [Pa] }\end{array}$ & $\begin{array}{c}\text { Tempo de } \\
\text { Abastecimento [s] }\end{array}$ & $\begin{array}{c}\text { Perda de carga } \\
\text { média [Pa] }\end{array}$ \\
\hline 1 & 500 & 0.188 & 367.5 \\
\hline 2 & 1000 & 0.199 & 761.9 \\
\hline 3 & 298.5 & 0.168 & 228.8 \\
\hline
\end{tabular}

\section{CONCLUSÃO}

Todas as indústrias, especialmente os fabricantes de automóveis, estão sempre procurando por soluções no desenvolvimento e aprimoramento de veículos. Hoje em dia, a utilização de simulações computacionais está se tornando uma prática comum nas indústrias. Considerando as melhorias de softwares e hardwares ao longo dos anos, o nível de complexidade das simulações computacionais está também em crescimento.

Hoje em dia, os códigos CFD possuem algoritmos mais poderosos e, junto com o aumento do número de processadores, análises multifásicas, que são simulações que consideram dois ou mais fluidos no mesmo volume de controle, vem se tornando uma excelente prática para o aumento da produtividade e redução do tempo de fabricação. Este fato faz com que o uso de simulações CFD aumentem entre os fabricantes de automóveis.

No presente trabalho, uma metodologia de avaliação de simulações multifásicas do processo de abastecimento de combustível de um veículo em condições reais, com uma vazão volumétrica de $50 \mathrm{l} / \mathrm{min}$, foi desenvolvida e apresentada. Um estudo complementar da pressão do tanque de combustível também foi avaliado. A simulação com uma curva de pressão do tanque de combustível medida a partir de teste físicos apresentou bons resultados de tempo de abastecimento e perda de carga.

Como estudo complementar, uma correlação do perfil de escoamento considerando todo o sistema de abastecimento de combustível com o duto de um material transparente, como o acrílico, seria interessante.

\section{REFERÊNCIAS}

1. Banerjee, R., Bai, X., Pugh, D., Isaac, K. et al., CFD Simulations of Critical Components in Fuel Filling Systems, SAE Paper, Society of Automotive Engineers, 2012.

2. Buscariolo, F.F.; Karbon, K.J., Comparative CFD Analysis Between Rotating and Static Cases of Different Wheels Opening Designs over a Performance Sedan, SAE Paper, $\mathrm{N}^{\circ}$ 2011-36-0271, Society of Automotive Engineers, 2011.

3. Buscariolo, F. F.; Volpe L. J. D., Water Ingestion and Pressure analysis of Automotive Vehicles using Multiphase CFD, SAE Paper, Society of Automotive Engineers, 2014. 
4. Buscariolo, F. F.; Volpe, L. J. D.; Alves, J. C. L., Water Ingestion and Pressure analysis of Automotive Vehicles using Multiphase CFD, SAE Paper, Society of Automotive Engineers, 2014.

5. FLUENT 14.0., User's Guide, Fluent Inc., 2011.

6. HUCHO, W. H., Aerodynamics of Road Vehicles, From Fluid Mechanics of Vehicle Engineering, 4th Edition, SAE International, 1998.

7. Hypermesh 11.0 User's manual Altair Computing, Inc., 2011.

8. KELLY, K. B.; PROVENCHER, L. G.; SCHENKEL, F. K., The General Motors Engineering Staff Aerodynamics Laboratory - A full Scale Automotive Wind Tunnel, SAE Paper, No 820371, Society of Automotive Engineers, 1982.

\section{INFORMAÇÕES DE CONTATO}

Felipe Magazoni: felipe.magazoni@gm.com

Filipe Fabian Buscariolo: filipe.buscariolo@gm.com

Flavio Maruyama: flavio.maruyama@gm.com

Julio Cesar Lelis Alves: julio.alves@gm.com

Leonardo José Della Volpe: leonardo.volpe@gm.com

\section{DEFINIÇÕES/ABREVIAÇÕES}

CFD Computational Fluid Dynamics

VOF Volume of Fluid 